\title{
DIVERSIDADE DE ABELHAS VISITANTES DAS FLORES DE Citrus EM POMARES DE LARANJEIRA E TANGERINEIRA ${ }^{1}$
}

\author{
EDUARDO TIGRE DO NASCIMENTO², RAQUEL PÉREZ-MALUF³, \\ ROSIMEIRE ALVES GUIMARÃES ${ }^{4}$, MARIA APARECIDA CASTELLANI ${ }^{5}$
}

RESUMO-A presença de abelhas em culturas de valor comercial, no período de florescimento, é importante não apenas para garantir a polinização cruzada, quando indispensável, mas também para incrementar a produção de frutos e sementes. Nessa perspectiva, o presente trabalho teve a finalidade de identificar as abelhas visitantes das flores em pomar de laranjeira (Citrus sinensis) e tangerineira (Citrus reticulata) em Salinas - MG. O trabalho foi conduzido na fazenda experimental Santa Isabel, da Escola Agrotécnica Federal de Salinas - MG, em pomares de laranjeira com as variedades, pera, baía e baianinha, e de tangerineira, variedade poncã. As coletas das abelhas visitantes das culturas da laranjeira e tangerineira foram feitas nas floradas principais, entre os meses de agosto e outubro. Foram coletadas as abelhas que visitaram as flores nos horários entre $06 \mathrm{~h}$ e $18 \mathrm{~h}$, totalizando 52 horas na laranjeira e 50 horas na tangerineira. No pomar de laranjeira, foram coletados 5.045 espécimes com riqueza de 12 espécies, e na tangerineira, 1.428 espécimes com 20 espécies. As espécies de abelhas de maior predominância e dominância em todos os horários amostrados foram Apis mellifera e Trigona spinipes na cultura da laranjeira e A. mellifera, T. spinipes e Tetragonisca angustula na cultura da tangerineira.

Termos para indexação: Apidae, Estrutura de comunidades, Rutaceae.

\section{DIVERSITY OF FLOWERS VISITING BEES OF Citrus IN SALINAS, STATE OF MINAS GERAIS}

\begin{abstract}
The presence of bees on cultures with commercial value during flowering is important to ensure the cross-pollination, when necessary, but also to increase the production of fruits and seeds. This work aimed to identify flowers' visiting bees on orange and tangerine orchards in Salinas - MG. The work was leaded on Santa Isabel experimental farm of the Federal Agro-technical School of Salinas - MG, on orange orchards (Citrus sinensis) with pear, bahia and baianinha varieties in the spacing of 5,0 x 6,0, and on tangerine orchards (C. reticulate) using poncã variety in the spacing of 5,0 x 5,0. The sampling of visiting bees from orange and tangerine orchards was made during the main flowerings, between August and October. The bees that visited the flowers between 06:00 a.m. and 06:00 p.m. were collected. 5045 bees were collected with richness of 12 species in orange and 1428 bees with richness of 20 species in tangerine. The species of bees with major predominance and dominance in all sampled times were Apis mellifera and Trigona spinipes (orange) and Apis mellifera, Trigona spinipes e Tetragonisca angustula (tangerine).

Index terms: Apidae, Community structure, Rutaceae.
\end{abstract}

\footnotetext{
${ }_{1}^{1}$ (Trabalho 072-10). Recebido em: 18-03-10. Aceito para publicação em: 19-11-2010. Apoio financeiro CAPES.

${ }^{2}$ Mestrando em Agronomia, Programa de Pós-Graduação em Agronomia, Universidade Estadual do Sudoeste da Bahia, Caixa Postal 95, 45.083-900, Vitória da Conquista-BA, Brazil. E-mail: agrontigre@yahoo.com.br.

${ }^{3}$ Dra. em Biologia do Comportamento, Departamento de Ciências Naturais, Universidade Estadual do Sudoeste da Bahia, Caixa Postal 95, 45.083-900, Vitória da Conquista-BA, Brasil, E-mail: raquelmaluf@uesb.edu.br

${ }^{4}$ Mestre em Agronomia, Escola Agrotécnica Federal de Salinas, Salinas-MG, Brazil. E-mail: rosigui@uai.com.br

${ }^{5}$ Dra. em Agronomia, Departamento de Fitotecnia e Zootecnia, Universidade Estadual do Sudoeste da Bahia, Caixa Postal 95, 45.083900, Vitória da Conquista-BA, Brasil. E-mail: cidinhacastellani@gmail.com
} 


\section{INTRODUÇÃO}

O gênero Citrus é representado por plantas de porte médio (arbóreo/arbustivo), flores brancas e aromáticas, e frutos tipo baga (SWINGLE, 1943). As plantas cítricas são originadas de locais úmidos, tropicais e subtropicais da Ásia, e foram introduzidas no Brasil nos primórdios do descobrimento, inicialmente na Bahia. Devido a sua excelente adaptação nesta região, em 1540, o plantio da laranjeira já se havia expandido desde o litoral brasileiro até o interior do País (ROSSETTI, 2001).

$\mathrm{Na}$ atualidade, a citricultura brasileira apresenta números expressivos que demonstram a grande importância econômica e social que a cultura oferece para a economia do País (AZEVÊDO, 2003). De acordo com o Instituto de Economia Agrícola - IEA (2010), o Brasil exportou, no ano de 2008, o equivalente a 2.067.761 (Mil US\$), em produtos manufaturados de laranjeira e 51.114 (Mil US\$) em produtos manufaturados de outros citros. Segundo dados do Instituto Brasileiro de Geografia e Estatística - IBGE (2010), a produção brasileira de laranjeira, na safra de 2009, foi de mais de 18 milhões de toneladas.

Um dos aspectos relacionados com a produtividade de uma cultura é a polinização, que garante a fecundação dos óvulos e a formação do fruto. Em plantas cítricas, pode ocorrer tanto a autopolinização quanto a polinização cruzada, onde o pólen é transportado por insetos, ou pelo vento (REUTHER et al., 1968; LIM, 2001). No entanto, o vento é considerado um agente polinizador de mínima importância, uma vez que o pólen dos citros é viscoso, aderente e bastante pesado (SOLER, 1996, citado por AZEVEDO; PIO, 2002). Dentre os insetos que visitam as flores de Citrus, a maioria é da ordem Hymenoptera, onde as abelhas são responsáveis por cerca de $80 \%$ dessas visitas (McGREGOR, 1976).

Pesquisas realizadas por Malerbo-Souza et al. (2003) revelaram que as flores de laranjeira, quando visitadas por abelhas, produziram maior quantidade de frutos, frutos mais pesados, mais doces e com maior número médio de sementes por gomo, quando comparadas a flores não visitadas pelas abelhas. Em toranja (C. paradisi), Chacoff e Aizen (2006) observaram que flores emasculadas e encobertas apresentaram frutificação inferior quando comparadas com as flores deixadas para livre polinização ou tiveram polinização manual.

O estudo da estrutura da comunidade de abelhas presentes nos pomares de citros é importante para delinear estratégias de manejo que favoreçam a permanência dos visitantes florais mais frequentes e constantes durante a florada e, assim, buscar o au- mento da produtividade da cultura. Neste contexto, o objetivo deste trabalho foi estudar a comunidade de abelhas (Hymenoptera: Apidae) visitantes das flores da laranjeira (Citrus sinensis L.) e da tangerineira (Citrus reticulata Osbeck), identificando as espécies com potencialidade para o manejo de polinizadores.

\section{MATERIAL E MÉTODOS}

O presente trabalho foi desenvolvido no período de floração de citros, nos meses de agosto a outubro de 2005, nas culturas de laranjeira e tangerineira, localizadas na Fazenda Experimental Santa Isabel, pertencente à Escola Agrotécnica Federal de Salinas, EAFSAL- MG. A Fazenda é situada na região norte de Minas Gerais, a uma altitude de 472 metros. Segundo a classificação climática de Köeppen, o tipo de clima predominante na região é o AW, quente e úmido - com estação chuvosa no verão. A temperatura média anual é de $25,2^{\circ} \mathrm{C}$. O município apresenta baixo índice de pluviosidade, com uma média anual de $700 \mathrm{~mm}$ de chuvas, sendo junho e julho os meses mais secos do ano (média de $7,45 \mathrm{~mm} / \mathrm{mês}$ ) e de novembro a março os meses mais chuvosos (média de 209,9 mm/mês). O solo em geral é bastante acidentado e de baixa fertilidade natural (SILVEIRA et al., 2002).

O campo experimental constou de uma área de aproximadamente 1,8 ha, cultivado com citros, com idade de 5 anos, sendo o pomar de laranjeira constituído pelas variedades Pera, Baianinha e Baía, com espaçamento de $5,0 \times 6,0 \mathrm{~m}$, totalizando 300 plantas, e o pomar de tangerineira, constituído pela cultivar Poncã, com espaçamento 5,0 x 5,0m, totalizando também 300 plantas. O pomar limitava-se com plantações de bananeira, goiabeira, cana-de-açúcar e com mata nativa de vegetação de caatinga.

As coletas foram realizadas em cada cultura, entre $06 \mathrm{~h}$ e $18 \mathrm{~h}$. Foram determinados seis intervalos de $02 \mathrm{~h}$, e a cada dia eram sorteados os horários de coleta das abelhas nas flores. Na cultura da laranjeira, foram realizadas 26 coletas, totalizando 52 horas e na tangerineira foram realizadas 25 coletas, totalizando 50 horas. As abelhas foram capturadas com rede entomológica, identificadas em nível de espécie e depositadas na Coleção de Abelhas do Laboratório de Biodiversidade do Semiárido, na Universidade Estadual do Sudoeste da Bahia.

$\mathrm{O}$ estudo da estrutura da comunidade de abelhas visitantes das flores de laranjeira e tangerineira foi realizado pela estimativa da riqueza de espécies (S), o índice de diversidade de Shannon (H'). Para determinar a contribuição de cada espécie 
na caracterização da comunidade, foram calculadas: a frequência relativa, a constância (calculada a partir do total de coletas contendo a espécie i em relação ao total de coletas. Classificam-se as espécies em constantes $-\mathrm{W}$, quando presentes em mais de $50 \%$ das coletas; em acessórias - Y, quando presentes entre $25 \%$ e $50 \%$ das coletas e em acidentais - $\mathrm{Z}$, quando presentes em menos de $25 \%$ das coletas), e as espécies dominantes, quando as frequências relativas fossem superiores a 1/S (SILVEIRA NETO et al., 1976).

A análise de similaridade entre as culturas de citros foi feita pelo cálculo do índice de similaridade de Morisita (KREBS, 1989), e os índices de diversidade, analisados pelo teste $t$ do Programa Past (HAMMER et al., 2001).

Para as espécies consideradas dominantes e constantes, com potencial para o manejo de polinizadores em citros, foram avaliados dois aspectos importantes: (1) a influência do horário de amostragem sobre as abelhas coletadas por meio de uma análise de variância, e (2) a sobreposição no horário de exploração dos recursos pelo índice de Pianka (KREBS, 1989).

\section{RESULTADOS}

A comunidade de abelhas nas duas espécies vegetais estudadas foi representada por 16 gêneros e 25 espécies, com um total de 6.473 espécimes. As espécies Centris analis Fabricius, Xylocopa suspecta Moure \& Camargo e Pseudoaugochlora sp. foram exclusivas na cultura da laranjeira, e as espécies Centris sp., Centris inermis Friese, Ceratina (Crewella) sp., Augochloropsis sp.1, Augochloropsis sp.2, Augochloropsis sp.3 e Pseudoaugochlora pandora (Smith) foram exclusivas na cultura da tangerineira, durante o período das amostragens (Tabela 1).

$\mathrm{Na}$ laranjeira, foram coletados 5.045 indivíduos, distribuídos em 12 espécies, havendo predominância da espécie Apis mellifera L., com 3.913 indivíduos sobre as 11 espécies nativas, representadas por 1.132 indivíduos (Tabela 1). A diversidade, estimada pelo índice de Shannon, correspondeu a 0,59. A. mellifera e Trigona spinipes (Fabricius) somam 4.991 indivíduos $(98,9 \%$ ) das abelhas coletadas, o que demonstra a importante contribuição destas espécies no manejo da laranjeira. Constatou-se uma única espécie acessória, Tetragonisca angustula (Latreille), contribuindo com $0,71 \%$ dos indivíduos coletados. As demais espécies foram consideradas acidentais, com um total de indivíduos coletados por espécies variando de um a quatro (Tabela 1).

$\mathrm{Na}$ tangerineira, foram coletados 1.428 indivíduos com riqueza de 20 espécies, havendo predominância da espécie $A$. mellifera, com 1.034 indivíduos, sobre as 19 espécies nativas representadas por 394 indivíduos (Tabela 1). A diversidade, estimada pelo índice de Shannon, correspondeu a 1,0. Na tangerineira, as espécies mais abundantes foram A. mellifera, Trigona spinipes e Tetragonisca angustula, que também foram classificadas como constantes e dominantes (Tabela 1). Estas três espécies contribuíram com $94,3 \%$ do total de indivíduos coletados. Não foi constatada a presença de espécies acessórias. As demais 17 espécies foram consideradas acidentais, com um total de indivíduos coletados por espécies variando de um a 16 (Tabela 1).

As duas culturas apresentaram similaridade alta, constatada pelo índice de Morisita igual a 0,99, devido principalmente à predominância de $A$. mellifera sobre as demais espécies, nas duas culturas. Entretanto, a cultura da tangerineira apresentou diversidade significativamente maior que a da laranjeira (teste $t=11,27 ; \mathrm{p}<0,001$ ).

\section{Horários de visitação}

As análises realizadas com as espécies dominantes e constantes de cada pomar apontam para uma diferença significativa na atividade de visitação das flores de laranjeira, tanto para $A$. mellifera $(\mathrm{F}=3,11 ; \mathrm{p}=0,03)$ quanto para Trigona spinipes $(\mathrm{F}=3,12 ; \mathrm{p}=0,04)$ (Figura 1). Para a tangerineira, não houve diferença significativa entre os horários de atividade das espécies $A$. mellifera $(\mathrm{F}=0,29)$, Tetragonisca angustula $(\mathrm{F}=1,81)$ e Trigona spinipes $(\mathrm{F}=1,58)$ (Figura 2).

Observou-se uma sobreposição nos horários de exploração dos recursos entre as espécies consideradas dominantes, nas duas culturas. O valor do índice de sobreposição de Pianka (KREBS, 1989) foi de 0,98 entre $A$. mellifera e $T$. spinipes no pomar de laranjeira (Figura 1). Já no pomar da tangerineira, os valores observados foram de 0,84 entre $A$. mellifera e $T$. spinipes, de 0,92 entre $A$. mellifera e Tetragonisca angustula e de 0,68 entre T. spinipes e Tetragonisca angustula (Figura 2).

\section{DISCUSSÃO}

Trabalhos relacionados aos visitantes florais de Citrus e sua provável contribuição à polinização são escassos. Estudos conduzidos avaliando a cultura da laranjeira (C. sinensis) e toranjeira (C. paradise) constaram que $A$. mellifera foi o visitante floral mais frequente nas flores (MALERBO-SOUZA et al., 2003; CHACOFF; AIZEN, 2006), corroborando os resultados apresentados neste estudo. MalerboSouza et al. (2003) observaram ainda que o néctar é 
o recurso mais coletado por estas abelhas nas flores da laranjeira $(94,40 \%)$.

A. mellifera é uma espécie exótica no Brasil, que compete com as espécies nativas na exploração dos recursos florais, sendo predominante na estrutura de diferentes comunidades já estudadas (AGUIAR; MARTINS, 2002; BIESMEIJER; SLAA, 2006; CARMO et al., 2004; GAMITO; MALERBOSOUZA, 2006, MALERBO-SOUZA et al., 2003). Outras espécies sociais, como Trigona spinipes e Tetragonisca angustula, foram consideradas dominantes neste estudo, mas a contribuição delas no percentual total de abelhas coletadas é baixa (aproximadamente $22 \%$ na laranjeira e na tangerineira). As abelhas solitárias constituíram grupos acidentais.

Essa predominância de $A$. mellifera reflete-se nos baixos índices de diversidade (0,59 e 1) estimados para as duas culturas. Pinheiro-Machado et al. (2002) estimaram índices de diversidade de abelhas entre 2,11 em áreas de caatinga. Em estudos realizados em cultivos de urucum (COSTA et al., 2008) e goiabeira (GUIMARÃES et al., 2009), ambos em região de caatinga, os índices de diversidade estimados foram de 1,58 e 0,72 , respectivamente.

No pomar de laranjeira, foi coletada maior abundância de abelhas, destacando-se A. mellifera e Trigona spinipes, em relação à cultura da tangerineira, o que não refletiu em maior riqueza de espécies e diversidade. Um fator que poderia explicar esse resultado é a abundância do número de flores presentes na laranjeira em relação às flores na tangerineira, que tornaria essa cultura mais atrativa para as abelhas. Durante as coletas, foi observado que as flores da laranjeira se distribuíam em toda a área da planta. Na cultura da tangerineira, as flores concentravam-se na região apical da planta. Essa diferença visualmente constatada na oferta dos recursos explorados pelas abelhas, provavelmente afeta o comportamento forrageiro dos visitantes florais (STONE, 1994; STEFFAN-DEWENTER; TSCHARNTKE, 2000; KLEIN et al., 2004; POTTS et al., 2004). Em geral, espera-se que plantas que disponibilizam maior quantidade de flores atraiam um número maior de potenciais polinizadores (SAKAI, 2002; FORSYTH, 2003).

Outro fator analisado é a presença de espécies eussocial nos dois pomares. Das 12 espécies coletadas na laranjeira, seis são sociais e, na tangerineira, das 20 espécies, oito são sociais (Tabela 1). As espécies sociais necessitam coletar néctar e pólen para seus ninhos frequentemente populosos e perenes, e recrutam outras campeiras (STEFFAN-DEWENTER; TSCHARNTKE, 2000). As espécies solitárias não apresentam o comportamento de recrutamento observado nas espécies sociais (MICHENER, 2000), o que resulta em um número menor de indivíduos que forrageiam.

As análises realizadas sobre os horários de visitação evidenciaram que, no pomar de laranjeira, A. mellifera apresentou um pico de abundância pela manhã, entre $8 \mathrm{~h}$ e $10 \mathrm{~h}$, e outro à tarde, entre $14 \mathrm{~h}$ e 16h. Trigona spinipes apresentou uma atividade mais intensa nos horários da manhã. Na tangerineira, $A$. mellifera apresentou uma oscilação na atividade de forrageio, Tetragonisca angustula iniciou suas atividades após as $8 \mathrm{~h}$, e Trigona spinipes manteve sua atividade constante ao longo do dia. Apesar de estas espécies explorarem os recursos simultaneamente, A. mellifera foi numericamente superior em todos os horários coletados nos dois pomares (Figuras 1 e 2). 
TABELA 1 - Total de abelhas coletadas nos dois agroecossistemas, Laranjeira (Citrus sinensis L.) e Tangerineira (C. reticulata Osbeck.) e análise faunística, Salinas - MG. N: número total de indivíduos; F: frequência relativa; C: constância, sendo constante (W), acessória (Y) e acidental (Z); D: dominância, sendo dominante $(\mathrm{D})$ e não dominante $(\mathrm{Nd})$.

\begin{tabular}{|c|c|c|c|c|c|c|c|c|}
\hline \multirow[b]{2}{*}{ Espécie } & \multicolumn{4}{|c|}{ Laranjeira } & \multicolumn{4}{|c|}{ Tangerineira } \\
\hline & $\mathbf{N}$ & $\mathbf{F}$ & $\mathbf{C}$ & D & $\mathbf{N}$ & $\mathbf{F}$ & $\mathbf{C}$ & D \\
\hline Apis mellifera $\mathrm{L}$. & 3913 & 0,7756 & W & $\mathrm{D}$ & 1034 & 0,7241 & $\mathrm{~W}$ & $\mathrm{D}$ \\
\hline Bombus (Fervidobombus) morio (Swederus) & - & & & & 1 & 0,0007 & $\mathrm{Z}$ & $\mathrm{Nd}$ \\
\hline Centris (Centris) inermis Friese & - & & & & 1 & 0,0007 & $\mathrm{Z}$ & $\mathrm{Nd}$ \\
\hline Centris (Heterocentris) analis (Fabricius) & 1 & 0,0002 & $\mathrm{Z}$ & $\mathrm{Nd}$ & - & & & \\
\hline Centris (Trachina) fuscata Lepeletier & 4 & 0,0008 & $\mathrm{Z}$ & $\mathrm{Nd}$ & 12 & 0,0084 & $\mathrm{Z}$ & $\mathrm{Nd}$ \\
\hline Centris sp. & - & & & & 1 & 0,0007 & $\mathrm{Z}$ & $\mathrm{Nd}$ \\
\hline Ceratina (Crewella) sp. & - & & & & 2 & 0,0014 & $\mathrm{Z}$ & $\mathrm{Nd}$ \\
\hline Euglossa sp.2 & 1 & 0,0002 & $\mathrm{Z}$ & $\mathrm{Nd}$ & - & & & \\
\hline Exomalopsis (Exomalopsis) analis Spinola & - & & & & 10 & 0,007 & $\mathrm{Z}$ & $\mathrm{Nd}$ \\
\hline Frieseomelitta doerdeleini (Friese) & 3 & 0,0006 & $\mathrm{Z}$ & $\mathrm{Nd}$ & 13 & 0,0091 & $\mathrm{Z}$ & $\mathrm{Nd}$ \\
\hline Frieseomelitta languida Moure & 3 & 0,0006 & $\mathrm{Z}$ & $\mathrm{Nd}$ & 16 & 0,0112 & $\mathrm{Z}$ & $\mathrm{Nd}$ \\
\hline Melipona quadrifasciata Lepeletier & - & & & & 1 & 0,0007 & $\mathrm{Z}$ & $\mathrm{Nd}$ \\
\hline Oxytrigona tataira Smith & 3 & 0,0006 & $\mathrm{Z}$ & $\mathrm{Nd}$ & 4 & 0,0028 & $\mathrm{Z}$ & $\mathrm{Nd}$ \\
\hline Tetragonisca angustula (Latreille) & 36 & 0,0071 & $\mathrm{Y}$ & $\mathrm{Nd}$ & 106 & 0,0742 & $\mathrm{~W}$ & $\mathrm{D}$ \\
\hline Trigona spinipes (Fabricius) & 1078 & 0,2137 & $\mathrm{~W}$ & $\mathrm{D}$ & 207 & 0,145 & $\mathrm{~W}$ & $\mathrm{D}$ \\
\hline Xylocopa (Neoxylocopa) grisescens Lepeletier & 1 & 0,0002 & $\mathrm{Z}$ & $\mathrm{Nd}$ & - & & & \\
\hline Xylocopa (N.) suspecta Moure e Camargo & 1 & 0,0002 & $\mathrm{Z}$ & $\mathrm{Nd}$ & - & & & \\
\hline Augochlora sp. & - & & & & 1 & 0,0007 & $\mathrm{Z}$ & $\mathrm{Nd}$ \\
\hline Augochloropsis sp. & - & & & & 2 & 0,0014 & $\mathrm{Z}$ & $\mathrm{Nd}$ \\
\hline Augochloropsis sp & - & & & & 3 & 0,0021 & $\mathrm{Z}$ & $\mathrm{Nd}$ \\
\hline Augochloropsis sp. & - & & & & 6 & 0,0042 & $\mathrm{Z}$ & $\mathrm{Nd}$ \\
\hline Augochloropsis sp. & - & & & & 2 & 0,0014 & $\mathrm{Z}$ & $\mathrm{Nd}$ \\
\hline Augochloropsis sp. & - & & & & 4 & 0,0028 & $\mathrm{Z}$ & $\mathrm{Nd}$ \\
\hline Pseudoaugochlora pandora (Smith) & - & & & & 2 & 0,0014 & $\mathrm{Z}$ & $\mathrm{Nd}$ \\
\hline Pseudoaugochlora sp. & 1 & 0,0002 & $\mathrm{Z}$ & $\mathrm{Nd}$ & - & - & - & - \\
\hline Total & 5045 & & & & 1428 & & & \\
\hline
\end{tabular}

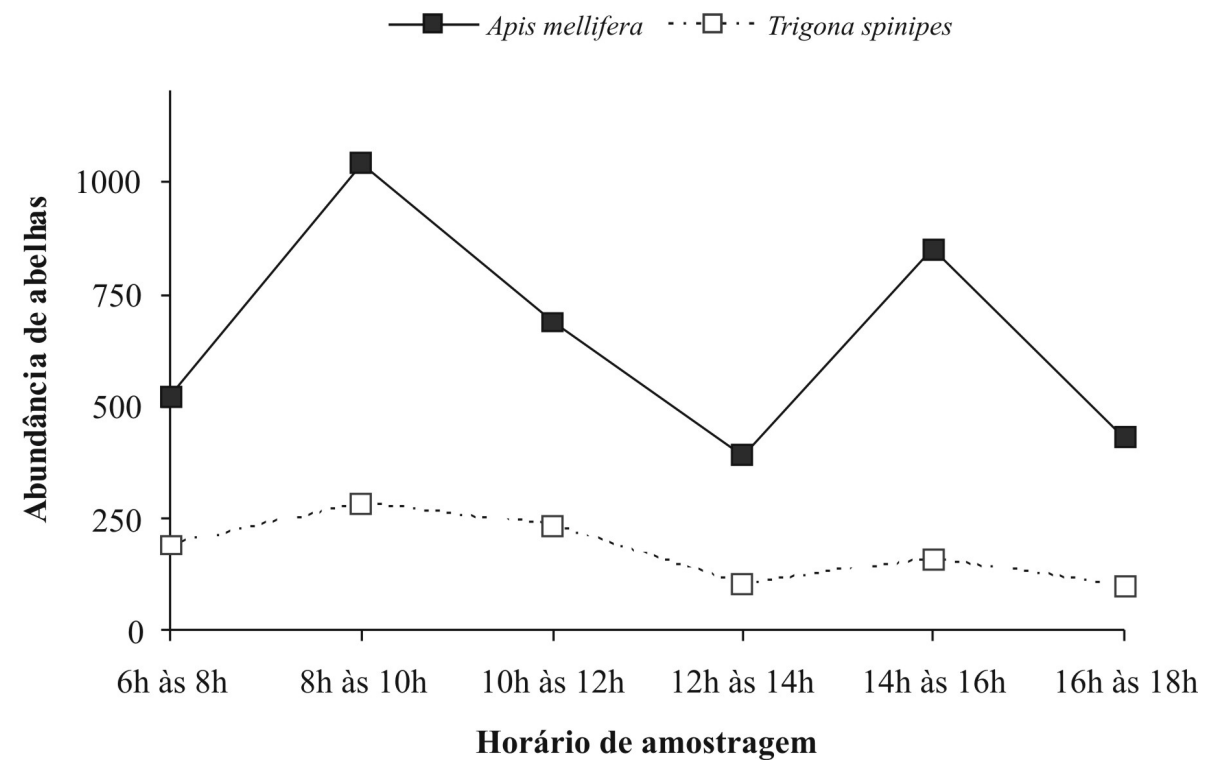

FIGURA 1 - Abundância de Apis mellifera L. e Trigona spinipes (Fabricius) coletadas nos diferentes horários de amostragem nas flores de Laranjeira (Citrus sinensis), Salinas-MG. 


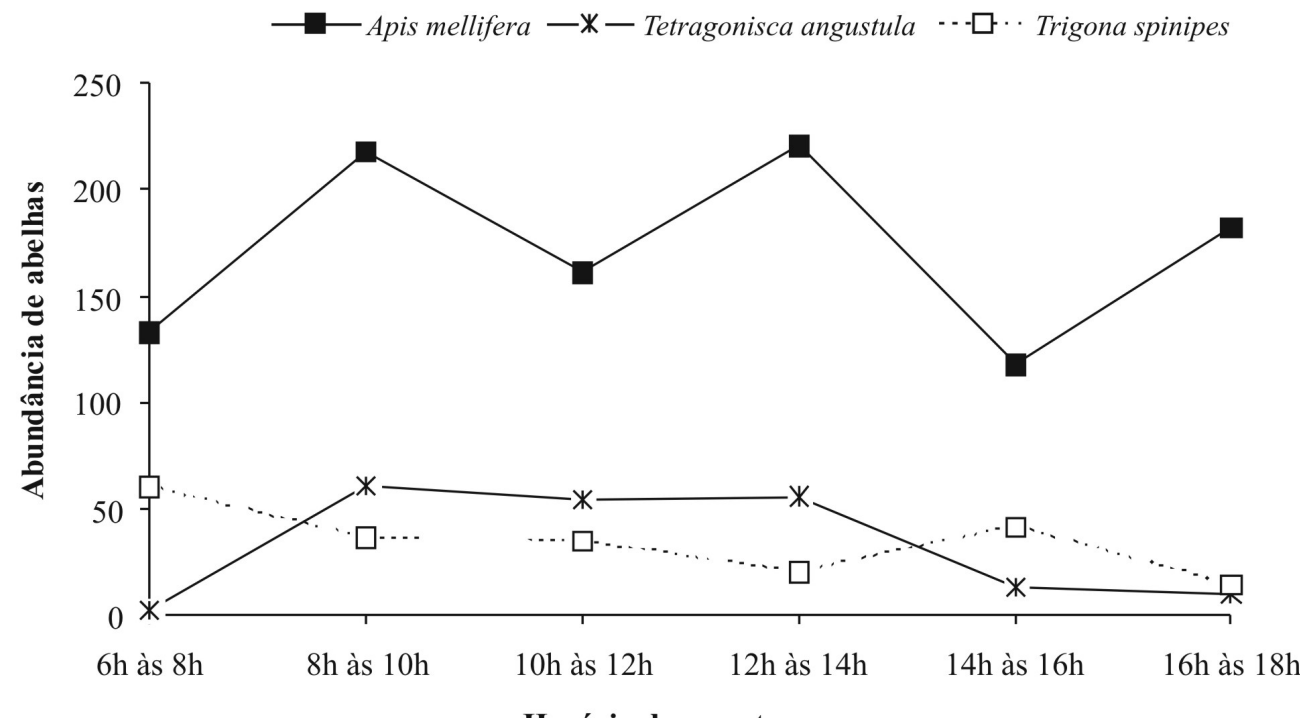

Horário de amostragem

FIGURA 2 - Abundância de Apis mellifera L., Tetragonisca angustula (Latreille) e Trigona spinipes (Fabricius) coletadas nos diferentes horários de amostragem nas flores de Tangerineira (Citrus reticulata), Salinas-MG.

\section{CONCLUSÕES}

Os resultados obtidos nos estudos realizados permitem concluir que Apis mellifera tem potencial polinizador em pomares comerciais de laranjeira e tangerineira. A atividade de forrageamento das abelhas nas flores de citros estende-se por todo o dia, o que deverá ser levado em consideração no planejamento das atividades de manejo da cultura, no período de floração

\section{REFERÊNCIA}

AGUIAR, A. J. C.; C. F. MARTINS. Abelhas e vespas solitárias em ninhos-armadilha na Reserva Biológica Guaribas (Mamanguape, Paraíba, Brasil). Revista Brasileira de Zoologia, São Paulo, v.19, p.101-116, 2002.

\section{AZEVÊDO, C.L.L.. Sistema de produção de citros} para o nordeste. EMBRAPA, 2003. Disponível em: $<$ http://sistemasdeproducao.cnptia.embrapa.br/ FontesHTML/Citros/CitrosNordeste/processamento. htm>. Acesso em: 27 jan. 2010.
AZEVEDO, F. A.; PIO, R. M. Estudo da polinização na redução do número de sementes do tangor 'Murcott'. Laranjeira, Cordeirópolis, v. 23, n. 2, p. 489-497, 2002.

BIESMEIJER, J.C.; SLAA, E. J. The structure of eusocial bee assemblages in Brazil. Apidologie Versailles, v.37, p.240-258, 2006.

CARMO, R.M.; FRANCESCHINELLI, E.V.; SILVEIRA, F.A. Introduced honeybees (Apis mellifera L.) reduce pollination success without affecting the floral resource taken by native pollinators. Biotropica, Belo Horizonte, v.36, n.3, p.371-376, 2004.

COSTA, A. J. C.; GUIMARÃES-DIAS, F.; PÉREZMALUF, R. Abelhas (Hymenoptera: Apoidea) visitantes das flores de urucum em Vitória da Conquista-BA. Ciência Rural, Santa Maria, v.38, n.2,p.534-537, 2008.

CHACOFF, N.P.;AIZEN, M.A. Edge effects on flower-visiting insects in grapefruit plantations bordering premontane subtropical forest. Journal of Applied Ecology, Oxford, 43, p. 18-27. 2006. 
FORSYTH, S.A. Density-dependent seed set in the Haleakala silversword: evidence for an Allee effect. Oecologia, Heidelberg, v.136, n.4, p.551-557, 2003.

GAMITO, L. M.; MALERBO-SOUZA, D. T. Visitantes florais e produção de frutos em cultura da laranja (Citrus sinensis L. Osbeck). Acta Scientiarum. Animal Sciences, Maringá, v.28, n.4, p.483488, 2006.

GUIMARÃES, R. A.; PÉREZ-MALUF, R.; Castellani M, A. Abelhas (Hymenoptera: apoidea) visitantes das flores de goiaba em pomar comercial in SalinasMG. Bragantia, Campinas, v.68, n.1, p.23-27, 2009.

HAMMER, O.; HARPER, D.A.T.; RYAN, P.D. Past: paleontological statistics software package for education and data analysis. Paleontologia Electronica, Califórnia, v.4, p.1-9, 2001.

IBGE - INSTITUTO BRASILEIRO DE GEOGRAFIA E ESTATÍSTICA. Tabela: produção, confronto das safras de 2008 e 2009 - Brasil. 2009. Disponível em: <http://www.ibge.gov.br/home/estatistica/indicadores/agropecuaria/lspa/lspa_200804_5.shtm >. Acesso em: 11 jan. 2010.

IEA - INSTITUTO DE ECONOMIA AGRÍCOLA. Área e produção dos principais produtos da agropecuária do Estado de São Paulo. Disponível em: $<$ http://www.iea.sp.gov.br/out/banco/menu.php>. Acesso em: 11 jan. 2010.

KLEIN, A.M.; STEFFAN-DEWENTER, I.; TSCHARNTKE, T. Foraging trip duration and density of megachilid bees, eumenid wasps and pompilid wasps in tropical agroforestry systems. Journal of Animal Ecology, Oxford, v.73, n.3, p.517-525. 2004.

KREBS, C. J. Ecological methodology. New York: Harper Collins, 1989. 654p.

LIM, A.L. Citrus and citroids: Recent biological findings. Malayan-Nature Journal, Kuala Lumpur, v.55 p.221-229. 2001.

MALERBO-SOUZA, D.T.; NOGUEIRA-COUTO, R.H.; COUTO, L.A. Polinização em cultura de laranjeira (Citrus sinensis L. Osbekc, var. Pera-rio). Brazilian journal of Veterinary Research and Animal Science, São Paulo, v.40 n.4, p.237-242, 2003.

McGREGOR, S.E. Insect pollination of cultivated crop plants. Washington: Agriculture Handbook, 1976. $411 \mathrm{p}$.
MICHENER, C. D. The bees of the world. Baltimore: The Johns Hopkins University Press, 2000. 913p.

PINHEIRO-MACHADO, C.; SANTOS, I. A. DOS; IMPERATRIZ-FONSECA, V. L.; KLEINERT, A. DE M. P.; SILVEIRA, F. A. DA. Brazilian bee surveys: state of knowledge, conservatios and sustainable use. In: KEVAN, P.; IMPERATRIZFONSECA, V. L. Pollinating bees: the conservation link between agriculture and nature. Brasília.: MMA, 2002. p.115-129.

POTTS, S.G.; VULLIAMY, B.; ROBERTS, S. Nectar resource diversity organises flower-visitor community structure. Entomologia Experimentalis Et Applicata, Dordrecht, v.113, n.2, p.103-107. 2004.

REUTHER, W.; BATCHELOR, L.;WEBBER, H. The citrus industry. Berkeley: University of California Press, 1968. v.2, 398p.

ROSSETTI, V. V. Manual ilustrado de doenças dos citros. São Paulo: Fealq/Fundecitrus, 2001. 207p.

SAKAI, S. General flowering in lowland mixed dipterocarp forests of southeast Asia. Biological Journal of the Linnean Society, London, v.75, n.2, p.233-247. 2002.

SILVEIRA, L.C.I.; BARBOSA, M.H.P.; OLIVEIRA, M.W. Manejo de variedades de cana-de-açúcar predominantes nas principais regiões produtoras de cachaça de Minas Gerais. Informe Agropecuário, Belo Horizonte, v.23, n.217, p.25-32, 2002.

SILVEIRA NETO. S.; NAKANO, O.; VILA NOVA, N. A. Manual de ecologia dos insetos. Piracicaba: Ceres, 1976. 419p.

STEFFAN-DEWENTER, J.; TSCHARNTKE, T. Resourse overlap and possible competition between honeybees and wild bees in central Europe. Oecologica, Paris, v.122, n.2, p.288-296, 2000.

STONE, G.N. Activity patterns of females of the solitary bee Anthophora plumipes in relation to temperature, nectar supplies and body size. Ecological Entomology, London, v.19, n.2, p.177-189, 1994.

SWINGLE, W.T. The botany of citrus and its wild relatives of the orange subfamily. In: WEBER, H.J., BATCHELLOR, L.D. The citrus industry. Berckley: University of California, 1943. v.1, p.129-474. 